\title{
Use of Cortically Fixed at Once Implants for the Treatment of Atrophic and Extreme Atrophic Jaws
}

\author{
Jimoh Olubanwo Agbaje ${ }^{1,2}$, Henri Diederich ${ }^{3 *}$ \\ ${ }^{1}$ Department of Imaging and Pathology, Catholic University Leuven, Belgium \\ ${ }^{2}$ St. John's Hospital, Belgium \\ ${ }^{3}$ Dental Clinic Henri Diederich, Luxembourg, UK
}

Submission: December 09, 2017; Published: February 08, 2018

*Corresponding author: Henri Diederich, Dental Clinic Henri Diederich, Luxembourg, UK, Tel: +352621144664; Email: hdidi@pt.lu

\section{Case Report}

\section{Summary}

Dentist and patient have limited treatment options for management of extremely atrophic jaws. The Cortically Fixed at Once implant system was developed to meet challenges in patients with severely resorbed jaw bones. The CF@O protocol requires no bone graft, no sinus lift or nerve displacements. The CF@0 implant system consists of several types of components specifically developed for different locations in the jaw. These components are Pterygoid implants, compressive implants and hybrid plates. The Pterygoid implants and the Hybrid plates are developed to be placed in the posterior zones of the maxilla. The compressive implants with specific macro- and micro-threads are used in the cortical and spongy bone, mostly in the frontal bone of the upper and lower jaw. The presented case reports describe the steps followed for the functional restoration of edentulous patients with the CF@O implant system.

Dentist and patient have limited treatment options for management of extremely atrophic jaws. The use of conventional intraosseous implants may be limited or inapplicable in restoring some edentulous spaces due to various anatomical reasons [1-3]. Even with the advent of implant dentistry a severely atrophied maxilla still constitutes a challenging therapeutic problem because advanced posterior alveolar resorption combined with increased maxillary sinus pneumatization often leaves insufficient bone for implant anchorage [4]. Bone augmentation is usually required to enable placement of a sufficient number and length of implants $[5,6]$.

The Cortically Fixed at Once (CF@0) implant system is designed to treat difficult cases. The CF@0 uses the remaining cortical bone to fix specially designed implants. This implant system was developed to meet challenges in patients with severely resorbed jaw bones. The CF@0 protocol requires no bone graft, no sinus lift or nerve displacements.

The CF@0 implant system consists of several types of components specifically developed for different locations in the jaw. These components are Pterygoid implants, compressive implants and hybrid plates. The Pterygoid implants and the Hybrid plates are developed to be placed in the posterior zones of the maxilla. The compressive implants with specific macro- and micro-threads are used in the cortical and spongy bone, mostly in the frontal bone of the upper and lower jaw. The hybrid titanium plates are thin, lightweight and highly flexible, which allows them to be adapted to any bone anatomy and fixed with osteosynthesis screws. The CF@0 protocol requires no graft, sinus lift nor nerve displacements.

\section{Components of CF@0}

The CF@0 implant system consists of several types of components specifically developed for different locations in the jaw. Pterygoid implants, compressive implants and hybrid plates. The Pterygoid implant and the hybrid plates are developed to be placed in the posterior regions of the maxilla. The compressive implants with specific macro- and micro-threads are used in the cortical and spongy bone. The hybrid titanium plates are thin lightweight and highly flexible which allows them to be adapted to any bone anatomy and fixed with osteosynthesis screws (Figure

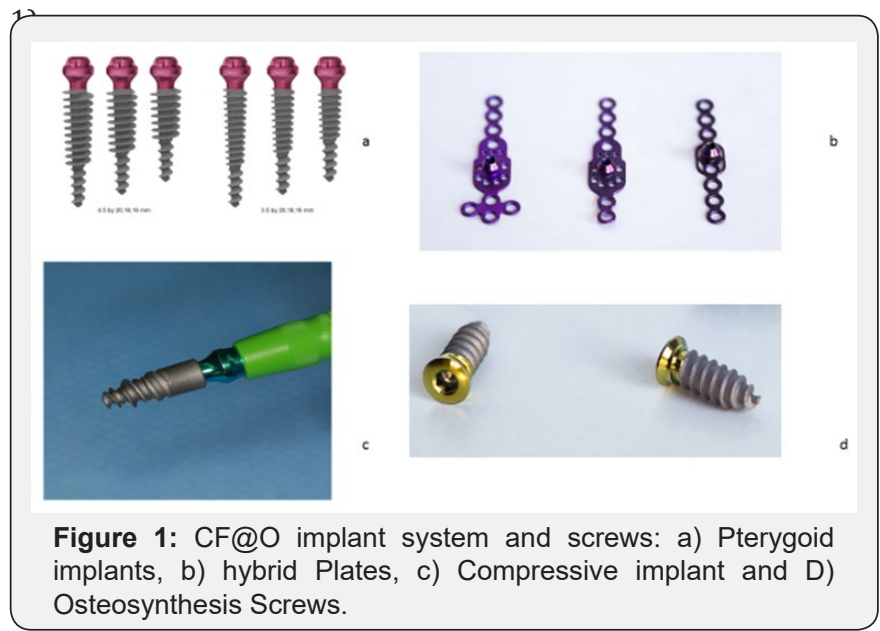

The following case reports describe procedures where CF@0 was used to rehabilitate edentulous jaws. 


\section{Advances in Dentistry \& Oral Health}

\section{Case presentations}

Patient 1 is a 65-year-old female who wanted fixed teeth in the right maxilla. Patient previously had an implant with delay loading and further management of the right maxilla required sinus lift before implant placement which the patient rejected. Patient was referred for further management. Clinical and radiographic examination showed severe vertical bone resorption in the right molar region (Figure 2). Patient was scheduled for pterygoid implant placement and a hybrid plate in position 16 .

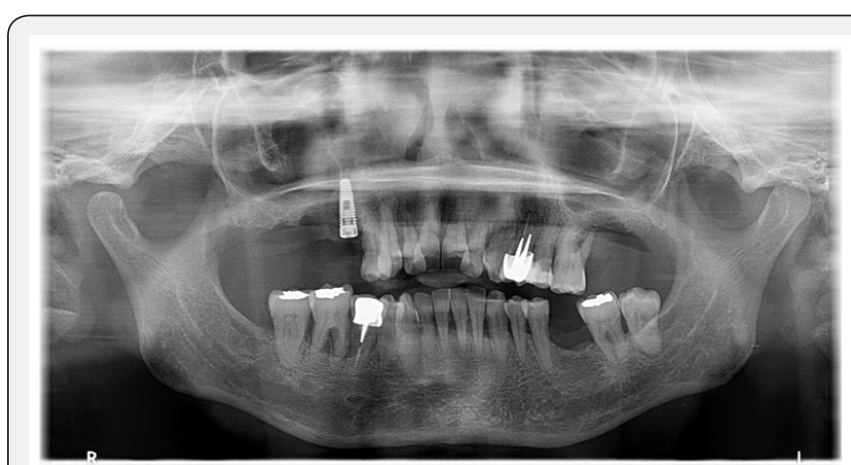

Figure 2: Panoramic radiograph of patient 1 at presentation.

At surgery, an open flap was made from the right tuberosity along the crest till the canine region in the maxilla. The flap was reflected on the vestibular side in positions 26 and 27 of the zygomatic arch; flap was also reflected in the palate. Pterygoid implant P3.5/20mm was inserted at the right pterygoid plate. One hybrid plate HENGG-2 (Highly efficient no graft gear) was fixed at position 16. The plate was fixed with osteosynthesis screws and covered with MatriboneR. After the implant placement, a silicone impression was taken. On the second appointment the framework was tried-in, and 10 days later a metal-ceramic bridge was delivered. A check-up was done 2 weeks later and then every 6 months afterward (Figure 3).

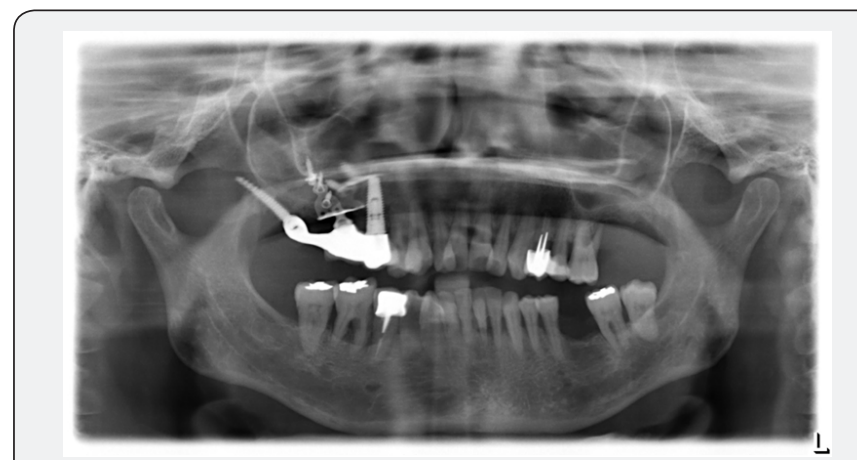

Figure 3: Panoramic radiograph of patient 1 at completion of the treatment.

Patient 2 is a 63-year-old non-smoker who wanted teeth fixed in the mandible. Patient presented with a complaint of mobile lower teeth. Clinical and radiographic examination showed gross mobility of lower teeth with generalised bone resorption (Figure 4). Patient was scheduled for total extraction of mandibular teeth and immediate implant placement in positions 47-37.

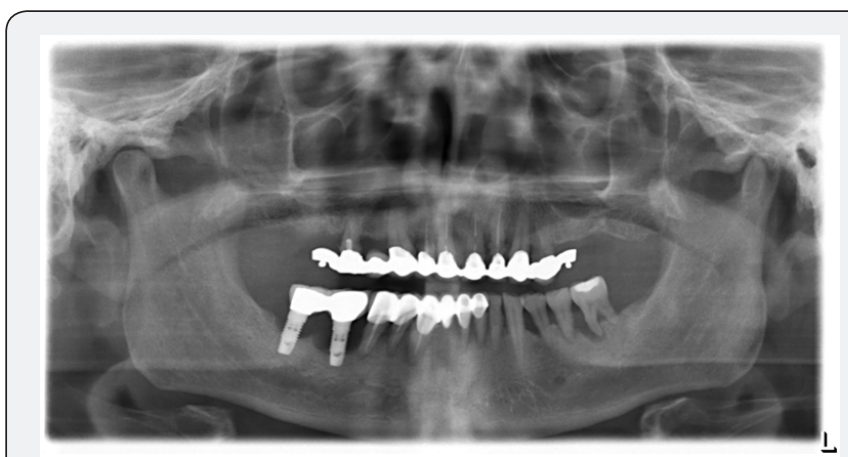

Figure 4: Panoramic radiograph of patient 2 at presentation.

At surgery, the mandibular bone was reshaped and debrided following total extraction; thereafter, one-piece implants of $4 \mathrm{~mm}$ diameter and height from 8 to $12 \mathrm{~mm}$ were inserted in positions 34,35 and 37. In positions 32, 33, 41 and 43, one piece implants of $3.5 \mathrm{~mm}$ diameter and $14 \mathrm{~mm}$ length were inserted with a torque of $50 \mathrm{~N}$.

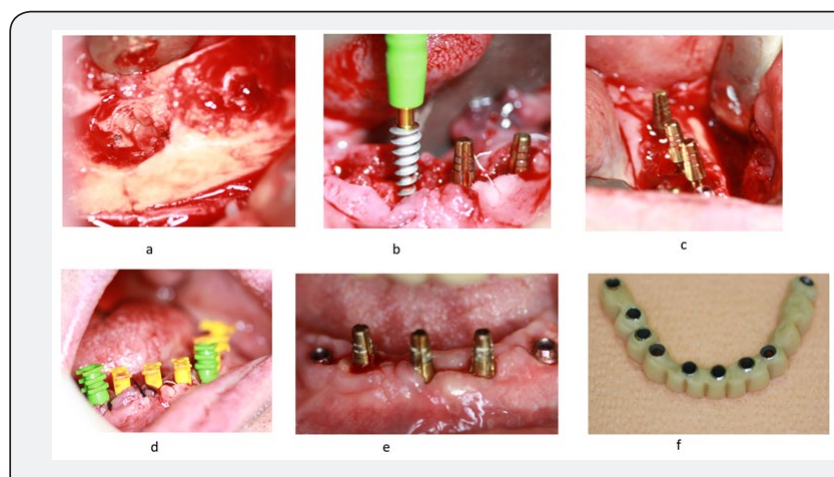

Figure 5: Surgical procedure and implant placement (a-e), metallic ceramic bridge (f).

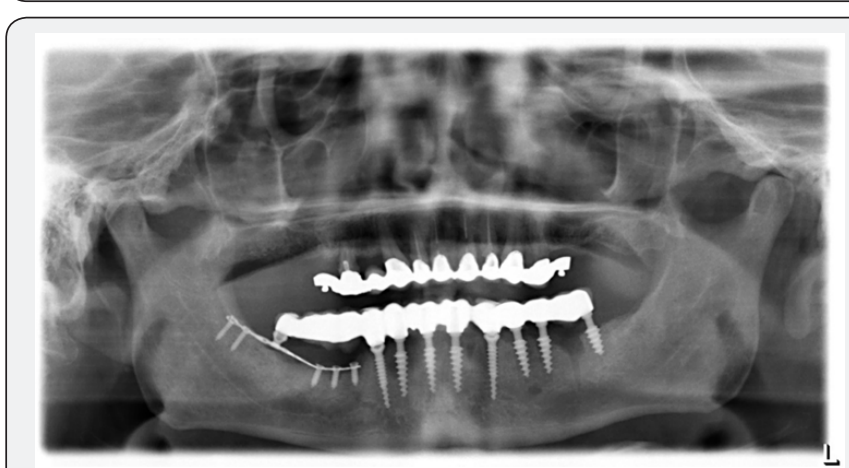

Figure 6: Panoramic radiograph of patient 2 at completion of the treatment.

The remaining gap between the implants and the extraction socket was fulfilled with grafting material (MatriboneR). Next, in the remaining mandibular bone, a hybrid plate HENGG-3 was installed and fixed with five osteosynthesis screws. After the implant placement, a silicone impression was taken with a transfer coping in place (Figure 5). A temporary resin bridge was made at the chairside and fixed on the mandible with temporary cement. Four days later the framework was tried-in, and 5 days 


\section{Advances in Dentistry \& Oral Health}

later a metal-ceramic bridge was delivered-half-screwed and halfcemented on the mandibular implant. A check-up was done 2 weeks later and then every 6 months afterward (Figure 6).

Patient 3 is a 54-year-old female, who presented at the clinic with a reason to get fixed teeth in the maxilla after wearing removable prosthesis for 25 years. A clinical examination showed a partial edentulous upper arch with a resorbed ridge, and a standing tooth with periodontal disease. Radiographic examination using an orthopantomogram showed an upper jaw with vertical bone resorption in the front and severe vertical resorption in the premolar and molar region (Figure 7). In the lower jaw several teeth were present in the frontal region.

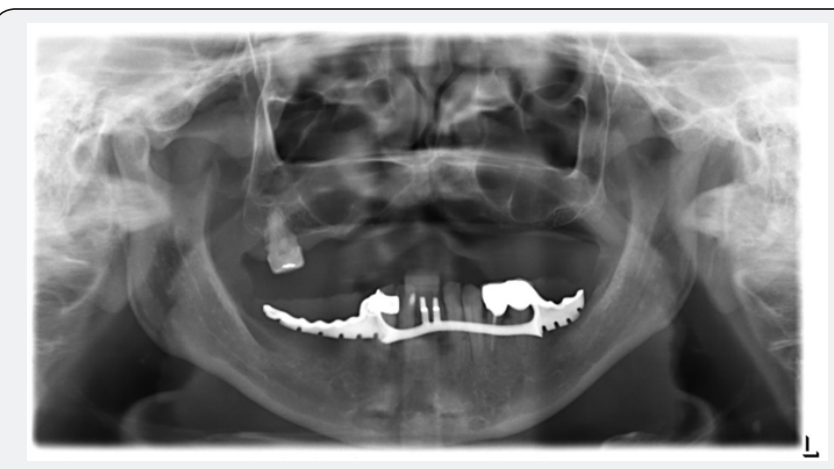

Figure 7: Panoramic radiograph of patient 3 at presentation showing edentulous upper arch with resorbed alveolus and remaining lower teeth.

\section{Treatment plan}

In the upper jaw, the treatment options are bone graft or sinuslift prior to implant placement. Patient rejected these treatment options and she preferred an immediate solution. An alternative plan was proposed, which is the placement of a combination of two pterygoid and four hybrid plates after the extraction of the remaining tooth. The patient agreed to this treatment plan.

In the maxilla, the remaining tooth was extracted and an open flap was made from the left tuberosity along the crest till the canine region. The flap was reflected on the vestibular side in positions 26 and 27 of the zygomatic arch; flap was also reflected in the palate. Pterygoid implant P3.5/20mm was inserted at the left pterygoid plate. Two hybrid plates HENGG-2 (Highly efficient no graft gear) were fixed at positions 23 and 25 . The plates were fixed with osteosynthesis screws and covered with MatriboneR. The procedure was quite similar for the right side. A plate HENGG-2 was installed in position 12 and another plate HENGG-1 at the zygomatic arch. The flap was then closed on the left and right with Poly Tetra Fluoro Ethylene Polymer (PTFE) monofilament non-absorbable suture. After an implant placement, the first bite registration was done with the old prosthesis of the patient. Then transfer coping was inserted and an impression was taken with silicone immediately after the surgery.

Four days after the framework, a try-in was done and a new bite registration was taken. Ten days after the try-in an appointment for prosthesis delivery was given. In the maxilla the metal acrylic bridge was screwed (Figure 8). The patient was reviewed after 2 weeks. Thereafter, patient was scheduled for follow-up at 3 months and then every 6 months.
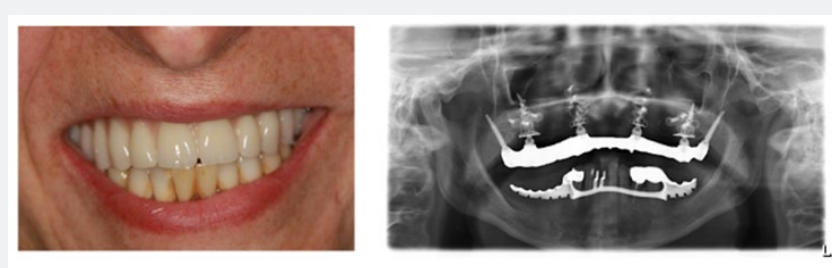

Figure 8: Panoramic radiograph of patient 3 at completion of treatment.

Keywords: Cortically Fixed at once implants; Pterygoid implants; Hybrid plates; Edentulous patient; Rehabilitation

\section{Discussion}

A severely atrophied maxilla presents limitations for conventional implant placement [1,5-8]. Dentistry now offers more options for management of atrophic jaw, including the use of various implant systems such as:

Eposteal/subperiosteal, endosteal, mini \& zygomatic implants, plus various regenerative grafting procedures [8-12]. Still some severe atrophic jaw cases defile these current treatment options.

Cortically fixed at Once implant system consists of specially designed plates suitable for extremely atrophic regions as well as screw implants of varying lengths to treat different difficult cases [13]. CF@0 uses the concept of tricortical support anchorage; it uses the available residual bone volume for support. The high fatigue strength of the solid titanium implants (without any welds or added parts) is particularly indicated for mechanically demanding situations (the canine and zygomatic sectors of the maxilla) and the mandibular ramus.

CF@O has a well-established protocol which allows immediate loading with the restoration of function and aesthetics within a few days [13]. In this way severe atrophies may be rehabilitated within 10 days with a fixed denture. This is a relief to patients who used to be rejected for implant treatment and for those who for many reasons cannot wait for months to have a complete treatment. Where traditional implant methods take several months to complete, the $\mathrm{CF} @ 0$ approach achieves the same result in days without the need for additional surgery.

The CF@0 approach incorporates the whole range of treatment procedures available to the implantologist and it is a reliable alternative to bone grafts in situations where there is substantial bone resorption. No bone graft and no more sinus lift for the rehabilitation in cases of atrophied maxilla and mandible are needed. The presented case reports describe steps followed for the functional restoration of an edentulous patient with CF@0 implant system. Rehabilitation of seemingly difficult edentulous cases was achieved within a short period of time. 


\section{References}

1. Ali SA, Karthigeyan S, Deivanai M, Kumar A (2014) Implant rehabilitation for atrophic maxilla: a review. J Indian Prosthodont Soc 14(3): 196-207.

2. Belser UC, Buser D, Hess D, Schmid B, Bernard JP, et al. (1998) Aesthetic implant restorations in partially edentulous patients--a critical appraisal. Periodontol 2000 17: 132-150.

3. Mericske-Stern RD, Taylor TD, Belser U (2000) Management of the edentulous patient. Clin Oral Implants Res 11(Suppl 1): 108-125.

4. Penarrocha-Oltra D, Candel-Marti E, Ata-Ali J, Penarrocha-Diago M (2013) Rehabilitation of the atrophic maxilla with tilted implants: review of the literature. J Oral Implantol 39(5): 625-632.

5. Jivraj S, Chee W, Corrado P (2006) Treatment planning of the edentulous maxilla. Br Dent J 201(5): 261-279.

6. Sevetz EB Jr (2006) Treatment of the severely atrophic fully edentulous maxilla: the zygoma implant option. Atlas Oral Maxillofac Surg Clin North Am 14(1): 121-136.

7. Bosse LP, Taylor TD (1998) Problems associated with implant rehabilitation of the edentulous maxilla. Dent Clin North Am 42(1): 117-127.

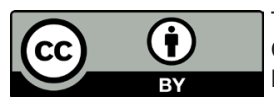

This work is licensed under Creative Commons Attribution 4.0 Licens DOI: 10.19080/ADOH.2018.07.555718
8. Candel E, Penarrocha D, Penarrocha M (2012) Rehabilitation of the atrophic posterior maxilla with pterygoid implants: a review. J Oral Implantol 38 Spec No: 461-466.

9. Cordaro L, Torsello F, Mirisola di Torresanto V, Baricevic M (2013) Rehabilitation of an edentulous atrophic maxilla with four unsplinted narrow diameter titanium-zirconium implants supporting an overdenture. Quintessence Int 44(1): 37-43.

10. Malo P, Nobre MA, Lopes I (2008) A new approach to rehabilitate the severely atrophic maxilla using extramaxillary anchored implants in immediate function: a pilot study. J Prosthet Dent 100(5): 354-366.

11. Mani V, Sivaprasad KK, George A, V Sankar Vinod, Miriam Mathew, et al. (2015) Hybrid Implant: A Novel Implant System. J Maxillofac Oral Surg 14(3): 720-727.

12. Sherry JS, Balshi TJ, Sims LO, Balshi SF (2010) Treatment of a severely atrophic maxilla using an immediately loaded, implant-supported fixed prosthesis without the use of bone grafts: a clinical report. J Prosthet Dent 103(3): 133-138.

13. Henri Diederich, Alexandre Junqueira Marques, Léo Guimarães Soares (2017) Immediate Loading of an Atrophied Maxilla Using the Principles of Cortically Fixed Titanium Hybrid Plates. Adv Dent \& Oral Health 3(3): 1-3.

\section{Your next submission with Juniper Publishers will reach you the below assets}

- Quality Editorial service

- Swift Peer Review

- Reprints availability

- E-prints Service

- Manuscript Podcast for convenient understanding

- Global attainment for your research

- Manuscript accessibility in different formats

( Pdf, E-pub, Full Text, Audio)

- Unceasing customer service

Track the below URL for one-step submission https://juniperpublishers.com/online-submission.php 